\title{
Cirugía en mayores de 80 años: Evolución clínica y costos asociados*
}

\author{
Drs. ATTILA CSENDES J. ${ }^{1}$, HANNS LEMBACH J. ${ }^{1}$, JUAN C. MOLINA F. ${ }^{1}$, \\ GONZALO INOSTROZA L. ${ }^{1}$, Int. STEPHANIE KÖBRICH S. ${ }^{2}$ \\ 1 Departamento de Cirugía, Hospital Clínico Universidad de Chile. \\ 2 Interna Facultad de Medicina Universidad de Chile. \\ Santiago, Chile.
}

\begin{abstract}
Surgical complications and costs among octogenarians

Background: Old age is a negative prognostic factor among patients subjected to surgical procedures. Aim: To assess the clinical profile and hospital stay costs among patients aged 80 years or more in a surgical department. Material and Methods: Retrospective review of medical records of 115 surgical patients aged $84 \pm 4$ years (67\% males), hospitalized during 2007. Clinical presentation, surgical treatment, functional status on admission and discharge and hospitalization costs, was analyzed. Results: Ninety percent of patients had associated diseases and $83 \%$ had previous surgical procedures. On admission, $88 \%$ of patients had a normal functional status. Hernia was the most common surgical diagnosis in $17 \%$. Thirty percent required emergency surgery. Mean hospital stay was 11 days, $47 \%$ required admission to the critical patients unit, $20 \%$ had complications, $8 \%$ required a second operation and two patients died. At discharge, $27 \%$ had a functional impairment. Hospitalization costs were 3.8 times greater among those that had complications, 3.1 times higher among those that required a second intervention and 1.8 times higher among those classified as III or IV according to American Surgical Association physical status classification, compared with those classified as I or II. Conclusions: Surgical patients aged more than 80 years, are more prone to complications and their hospitalization costs are higher.
\end{abstract}

Key words: Octogenarian, surgery, complications.

\section{Resumen}

Introducción: Los octogenarios son un grupo demográficamente emergente, que han generado cambios en la epidemiología del paciente quirúrgico, asociándose a mayores tasas de complicaciones, mortalidad y costos de atención. Objetivo: Estudiar el perfil clínico y costos de hospitalización de los pacientes octogenarios atendidos el año 2007 en nuestro departamento de cirugía. Material y Método: Revisión retrospectiva de los registros clínicos de pacientes quirúrgicos mayores de 80 años durante el año 2007. Se analizó la presentación clínica, tratamiento quirúrgico, evolución postoperatoria y status funcional (escala KATZ) al ingreso y alta.

* Recibido el 3 de Marzo de 2010 y aceptado para publicación el 4 de Mayo de 2010.

Correspondencia: Dr. Attila Csendes J.

Santos Dumont 999, Santiago, Chile.

E-mail: acsendes@redclinicauchile.cl 
Se analizó además los costos asociados a la hospitalización y tratamiento. Resultados: Serie de 115 pacientes con edad promedio de 83,7 años. Un $89,5 \%$ presentaron comorbilidades y 85,2\% cirugías previas. Al ingreso un $87,7 \%$ eran autovalentes (KATZ A-B). El diagnóstico quirúrgico más frecuente fue Hernia (16,87\%). Un $29,5 \%$ se intervino de urgencia. La estadía total promedio fue 10,6 días, requiriendo un 47\% de los pacientes Unidad de Pacientes Críticos (UPC). Un 20\% sufrió complicaciones (principalmente delirium), 7,8\% requirió reintervención, y dos pacientes fallecieron. Un 26,7\% presentó deterioro funcional al alta. El costo de hospitalización fue 3,75 veces mayor en los complicados, 3,1 veces mayor en los re-operados, 3,69 veces mayor en los que requirieron UPC, y 1,77 veces mayor en los ASA III-IV respecto a los I-II. Conclusión: Las complicaciones fueron en su mayoría no-quirúrgicas asociándose a hospitalizaciones prolongadas y de alto costo económico, con alta tasa de permanencia en UPC. Los factores asociados a mayor costo fueron complicación postoperatoria, re-operación, estadía prolongada en UPC y ASA III-IV.

Palabras clave: Octogenarios, cirugía, análisis de costos.

\section{Introducción}

Los pacientes octogenarios son un grupo demográficamente emergente. La proyección de crecimiento poblacional publicado por el Instituto Nacional de Estadísticas (INE) en base al censo de 2002 sitúa a este grupo como el de mayor crecimiento proporcional, con lo que llegaría el 2050 a representar un $6,87 \%$ de la población chilena $(1.388 .136 \text { personas })^{1}$. En el mundo desarrollado, y más recientemente en Chile, la transición demográfica ha generado un cambio en la epidemiología del paciente quirúrgico. Se observa actualmente en los servicios de cirugía un aumento de la proporción de pacientes ancianos ${ }^{2}$, con mayor presencia de patologías quirúrgicas más frecuentes en la tercera edad.

Como consecuencia de lo anterior, existe actualmente un aumento en la complejidad de los pacientes que requieren cirugía, dado por la mayor presencia de comorbilidades propias del adulto mayor, y que conllevan un aumento en el riesgo quirúrgico ${ }^{2}$. En este sentido estudios nacionales y extranjeros han demostrado la mayor susceptibilidad a complicaciones y mortalidad observadas en los adultos mayores cuando son sometidos a cirugía electiva y de urgencia $^{3,4}$. Esta tendencia genera hospitalizaciones más prolongadas, mayor requerimiento de camas de alta complejidad y mayor necesidad de procedimientos y reoperaciones, hecho que eleva los costos en el manejo de estos pacientes.

La consideración del aumento progresivo de este grupo etario en la actividad quirúrgica, además de la ya demostrada mayor dificultad y elevación de costos asociada a su manejo, nos obliga a conocer las características del comportamiento de este grupo específico al ser sometido a cirugía.

El objetivo de este trabajo es analizar el perfil clínico y los costos de hospitalización de los pacientes octogenarios atendidos en el Departamento de Cirugía del Hospital Clínico Universidad de Chile durante el año 2007.

\section{Material y Método}

Se revisaron retrospectivamente los registros clínicos de 115 pacientes quirúrgicos mayores de 80 años atendidos durante el año 2007 en el Hospital Clínico de la Universidad de Chile. Se analizó la presentación clínica, diagnóstico y tratamiento quirúrgico, además de la evolución postoperatoria considerando tiempo de hospitalización, uso de Unidad de Pacientes Críticos (UPC), y el status funcional al ingreso y alta.

Para medir el status funcional de los pacientes se usó la escala KATZ para actividades de la vida diaria, usada en geriatría. Se compone de 8 categorías de la A hasta la $\mathrm{H}$, en la cual el paciente se clasifica de acuerdo a su grado de dependencia de terceros para la alimentación, continencia, movilidad, uso del baño, vestirse y bañarse. La escala fue aplicada de acuerdo a antecedentes clínicos encontrados en la ficha al momento del ingreso y del alta.

Para evaluar el riesgo perioperatorio de cada paciente se utilizó la escala ASA de la Sociedad Americana de Anestesiología. Esta clasificación, usada ampliamente por anestesia, sitúa a los pacientes en una de 5 categorías ( $\mathrm{I}$ a V), de acuerdo a la presencia de comorbilidades y su estado de compensación al momento de la cirugía. Además agrega una letra E si se trata de un procedimiento de urgencia. La clasificación de cada paciente fue obtenida de la evaluación preoperatoria realizada por el anestesista encargado.

Se analizó además los costos asociados a la hospitalización y tratamiento de estos pacientes. Los resultados fueron tabulados usando Microsoft Excel®.

\section{Resultados}

La serie quedó compuesta por 115 pacientes, 38 mujeres y 77 hombres (33 y 67\%, respectivamente), con una edad promedio de 83,7 $\pm 3,69$ años. El 
$89,5 \%$ presentaba por lo menos una comorbilidad al ingreso, siendo la más frecuente la hipertensión arterial con $67,8 \%$, seguida de diabetes mellitus, con $17,4 \%$. El detalle de las comorbilidades se detalla en la Tabla 1 .

El $85,2 \%$ de los pacientes tenía antecedentes quirúrgicos, siendo la colecistectomía abierta y la apendicectomía los más frecuentemente realizados ( 28,7 y $26,1 \%$, respectivamente). El detalle de los antecedentes quirúrgicos se detalla en la Tabla 2.

El 29,5\% de los pacientes fueron sometidos a procedimientos quirúrgicos de urgencia, siendo las principales causas obstrucción intestinal $(27,2 \%)$, isquemia aguda de extremidades inferiores $(21,2 \%)$, y hernias complicadas $(18,1 \%)$. El 70,5\% restante correspondió a procedimientos electivos, realizándose principalmente hernioplastías $(20,1 \%)$, colecistectomía laparoscópica $(17,2 \%)$ y colecistectomía abierta (7,4\%). Los diagnósticos de los pacientes sometidos a cirugía de urgencia se resumen en la Tabla 3.

En la evaluación preoperatoria los pacientes con índice de ASA 1 fueron el 6,2\%, ASA 2 el 65,2\%, ASA 3 el 26,8\% y ASA 4 el 1,8\%. En el caso de los pacientes operados de urgencia, un 50\% fueron ASA 2 y un $40,6 \%$ ASA 3, mientras que los sometidos a cirugía electiva, un $71 \%$ eran ASA 2 y un $21 \%$ ASA 3. No se intervinieron en forma electiva pacientes ASA 4 o mayor.

El tiempo de hospitalización promedio fue de 10,6 días con un rango entre 1 y 173 . La tasa de complicaciones fue de $20 \%$. La más frecuente fue el delirium (6\%) seguida por la sepsis abdominal $(3,4 \%)$. Al separar por grupo, la tasa de complicación de los pacientes intervenidos de urgencia alcanza un $41,2 \%$, cifra significativamente mayor al $11,2 \%$ de los pacientes intervenidos en forma electiva. El detalle de las complicaciones postoperatorias se detalla en la Tabla 4.

El 7,8\% de los pacientes requirió una segunda

Tabla 1. Comorbilidades

\begin{tabular}{lc}
\hline Comorbilidades & \% \\
\hline HTA & 67,8 \\
Diabetes mellitus & 17,4 \\
Cardiopatía coronaria & 7,8 \\
ICC & 6,1 \\
Arritmias & 9,6 \\
Total pacientes con comorbilidades & 89,5 \\
\hline
\end{tabular}

HTA: Hipertensión Arterial, ICC: Insuficiencia Cardíaca Congestiva. intervención, en su mayoría por úlceras y pie diabético que requirieron manejo seriado. En sólo 2 de los pacientes que debieron ser reintervenidos, la indicación estuvo dada por una complicación quirúrgica (sepsis abdominal). El 47\% debió ser hospitalizado en unidades de pacientes críticos, con un promedio de 9,5 días y un rango entre 1 y 173 días. La tasa de mortalidad hospitalaria fue de $1,74 \%$, correspondiente a 2 pacientes: uno falleció por un infarto intestinal masivo secundario a una trombosis mesentérica y el otro, que fue intervenido por un síndrome de retención gástrica secundaria a un cáncer gástrico realizándose una gastrectomía total y esófago-yeyuno anastomosis termino lateral, evolucionó con una fístula de la anastomosis y falleció por una sepsis de foco abdominal.

Al ingreso, el 87,7\% de los pacientes eran autovalentes, es decir con índice de Katz A o B, sin embargo, al alta, este porcentaje disminuye a 75,4\%. El $26,7 \%$ de los pacientes presentó algún deterioro funcional al alta con disminución en la escala de Katz. El detalle de las evaluaciones del índice de Katz al ingreso y al alta se detalla en la Figura 1.

Tabla 2. Antecedentes Quirúrgicos

\begin{tabular}{lc}
\hline Antecedentes quirúrgicos & \% \\
\hline Apendicectomía & 26,1 \\
Colecistectomía abierta & 28,7 \\
Hernias & 19,1 \\
Cirugía urológica & 16,5 \\
Cirugía ginecológica & 16,5 \\
Total & 85,2 \\
\hline
\end{tabular}

Tabla 3. Diagnóstico post operatorio en los pacientes intervenidos de urgencia

\begin{tabular}{lcc}
\hline Diagnóstico & $\mathbf{n}$ & $\mathbf{\%}$ \\
\hline Obstrucción intestinal & 9 & 27,2 \\
Isquemia aguda EEII & 7 & 21,2 \\
Hernia complicada & 6 & 18,1 \\
Apendicitis aguda & 3 & 9,1 \\
Colecistitis aguda & 2 & 6,1 \\
Filtro de vena cava & 2 & 6,1 \\
Gangrena de Fournier & 1 & 3 \\
Otros & 3 & 9,1 \\
Total & 33 & 100,00 \\
\hline
\end{tabular}


CIRUGÍA EN MAYORES DE 80 AÑOS: EVOLUCIÓN CLÍNICA Y COSTOS ASOCIADOS

Tabla 4. Complicaciones postoperatorias en pacientes electivos y de urgencia

\begin{tabular}{|c|c|c|c|c|c|c|}
\hline \multirow{2}{*}{$\begin{array}{l}\text { Complicaciones } \\
\text { Complicados }\end{array}$} & \multicolumn{2}{|c|}{ Urgencia } & \multicolumn{2}{|c|}{ Electivo } & \multicolumn{2}{|c|}{ Total } \\
\hline & 14 & $41,2 \%$ & 9 & $11,2 \%$ & 23 & $20 \%$ \\
\hline Delirium & 2 & $5,8 \%$ & 5 & $61,7 \%$ & 7 & $6 \%$ \\
\hline Sepsis abdominal & 3 & $8,8 \%$ & 1 & $12,3 \%$ & 4 & $3,4 \%$ \\
\hline infección herida operatoria & 1 & $2,9 \%$ & 0 & $0 \%$ & 1 & $0,8 \%$ \\
\hline IRA & 1 & $2,9 \%$ & 0 & $0 \%$ & 1 & $0,8 \%$ \\
\hline TEP & 1 & $2,9 \%$ & 0 & $0 \%$ & 1 & $0,8 \%$ \\
\hline TVP & 0 & $0 \%$ & 1 & $12,3 \%$ & 1 & $0,8 \%$ \\
\hline Otras no quirúrgicas & 6 & $17,6 \%$ & 2 & $24,6 \%$ & 6 & $5,2 \%$ \\
\hline No complicados & 20 & $58,8 \%$ & 72 & $88,8 \%$ & 92 & $80 \%$ \\
\hline
\end{tabular}

IRA: Insuficiencia Renal Aguda, TEP: Tromboembolismo pulmonar, TVP: Trombosis venosa Profunda.

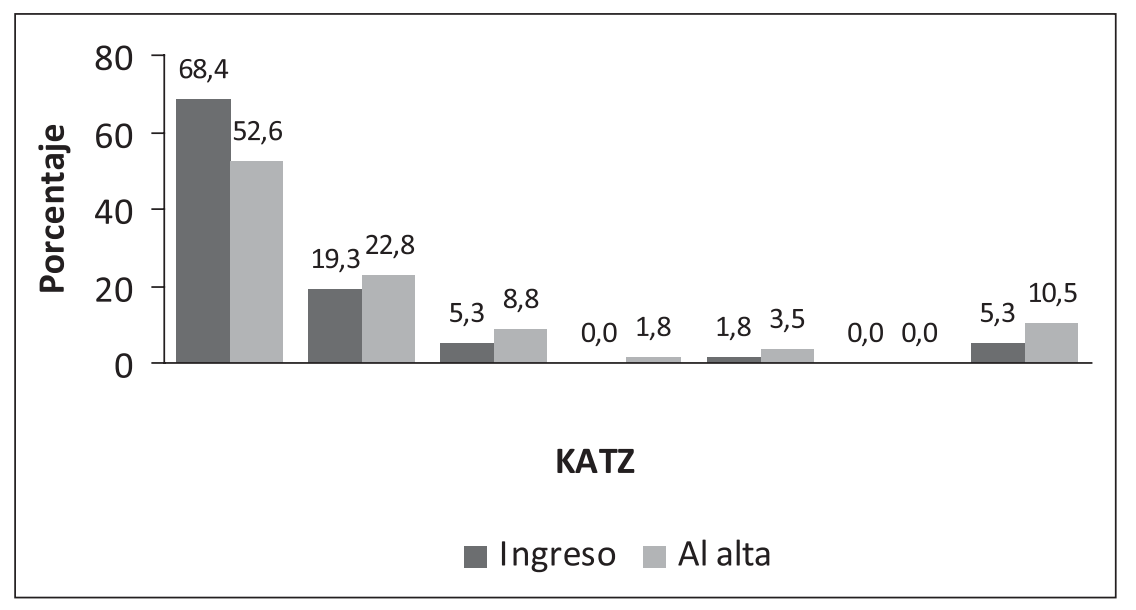

Figura 1. Índice de KATZ al ingreso y al alta.
Respecto a los costos de hospitalización, estos alcanzaron un promedio de $\$ 1.790 .733$ (pesos chilenos) por paciente, equivalente a U\$3.000, para aquellos que tuvieron una evolución sin complicaciones, comparado con un promedio de \$7.817.299 por paciente (US14.200), para aquellos que presentaron alguna complicación postoperatoria, significando un costo 3,75 veces superior. Al revisar el grupo que requirió reoperación durante su evolución, el costo promedio fue de $\$ 2.555 .350$ para los que no requirieron reintervención comparado con un promedio de \$7.915.393 para los reoperados, significando un costo 3,1 veces superior. Respecto a los pacientes que fueron admitidos a la unidad de pacientes críticos, el costo promedio fue de $\$ 4.960 .264$ para los que requirieron Unidad de Cuidados intensivos o intermedios, comparado con un costo promedio de $\$ 1.345 .143$ para los que no ingresaron a esta uni- dad, significando un costo 3,69 veces mayor en los primeros. Finalmente, al analizar por riesgo perioperatorio, los pacientes clasificados como ASA I-II tuvieron un costo promedio de hospitalización de $\$ 2.473 .106$, comparado con los \$4.384.465 que promediaron los pacientes ASA III-IV, representando un costo 1,77 veces mayor en estos últimos.

\section{Discusión}

Los cambios demográficos, los avances en cuidados perioperatorios y cirugía mínimamente invasiva, han ampliado las indicaciones quirúrgicas a pacientes progresivamente más ancianos y con comorbilidades complejas. Además de un riesgo quirúrgico mayor, las características fisiológicas, farmacológicas, y sociales de este grupo de pacientes 
son distintas a las de pacientes más jóvenes, y deben ser consideradas por el equipo quirúrgico, si se quiere mantener los resultados quirúrgicos actuales en el futuro, en el momento de la indicación de la cirugía $\mathrm{y}$ en el manejo perioperatorio ${ }^{2}$. Los octogenarios como grupo son pacientes con peor status funcional basal, con comorbilidades más complejas, y con mayor tasa de cirugía de urgencia que sus pares de menor edad ${ }^{5}$. Aunque clásicamente se han limitado las indicaciones de tratamiento quirúrgico en este grupo etario, la evidencia disponible no demuestra peores resultados en forma sistemática haciendo difícil para el cirujano la decisión de operar en cada caso particular. ${ }^{5}$

La edad como factor de riesgo quirúrgico independiente no se ha demostrado sistemáticamente en todas las publicaciones ${ }^{2}$. Aunque la mortalidad postoperatoria general aumenta en forma proporcional a la edad del paciente, análisis multivariados han dado mayor peso a la presencia y severidad de las comorbilidades más que a la edad misma ${ }^{5}$. Es así como un paciente octogenario puede tener menor riesgo que un paciente más joven portador de insuficiencia cardíaca.

Respecto a los procedimientos quirúrgicos realizados, destacan como más frecuentes el tratamiento quirúrgico de las hernias y de la obstrucción intestinal, además de la colecistectomía, todas patologías más prevalentes en grupos de mayor edad. Una publicación nacional ${ }^{4}$, que estudia cirugía abdominal de urgencia en adultos mayores, también destaca la patología de la pared abdominal como primera en frecuencia de indicación, hecho también evidenciado en publicaciones extranjeras. Análisis multivariados de factores de riesgo en estos pacientes han mostrado que el riesgo quirúrgico depende más de las condiciones del paciente y la oportunidad de la cirugía (urgente o no), que del tipo de cirugía a realizar³.

La tasa de complicaciones presentada en nuestra serie es de $19,1 \%$, similar a lo evidenciado en otras experiencias extranjeras que muestran tasas de complicaciones en torno a $20 \%$. Sin embargo, es necesario considerar que los grupos estudiados muchas veces difieren respecto a la inclusión de pacientes sometidos a cirugías de urgencia, la edad de corte y los tipos de cirugía. Es por esto que la interpretación de la tasa de mortalidad presentada en este trabajo $(1,7 \%)$ debe ser hecha atendiendo a las características de nuestra población estudiada. Por ejemplo, en un trabajo publicado en 2005, en que se estudiaron los resultados quirúrgicos en pacientes mayores de 80 años sometidos a cirugía no cardíaca se obtuvo una tasa de mortalidad general de $8 \%$, sin embargo, al hacer el análisis por tipo de cirugía, para la gran mayoría de los procedimientos quirúrgicos realizados la mortalidad era inferior a $2 \%$, pero para las cirugías más complejas las tasas de mortalidad ascendían hasta $60 \%$ en algunos casos, como en las lobectomías pulmonares ${ }^{5}$. En otro trabajo australiano publicado en 2007 se evidenció una mortalidad de $6 \%$ y morbilidad de $20 \%$, sin embargo, nuevamente la mayor parte de la mortalidad se atribuye a cirugías complejas (torácica, vascular arterial) ${ }^{3}$. En nuestra serie una proporción menor de pacientes fue sometida a cirugías de mayor complejidad, siendo la reparación de hernias y la colecistectomía laparoscópica los procedimientos más realizados. Sólo 1 paciente fue sometido a cirugía torácica y 4 a cirugía vascular. Esto podría explicar la baja tasa de mortalidad encontrada. Además, la mayor parte de nuestros pacientes fueron sometidos a cirugía electiva, que según lo reportado en la literatura tendría menor tasa de complicaciones y mortalidad respecto de la cirugía de urgencia ${ }^{6}$.

La clasificación ASA usada en anestesia ha mostrado en distintas publicaciones que consideran pacientes añosos, una directa relación con las tasas de complicación y mortalidad ${ }^{3,7}$. En nuestro trabajo esto además se relacionó a mayores costos de hospitalización, siendo el gasto 2,03 veces mayor en pacientes ASA III-IV respecto a los ASA I-II. Consideramos importante destacar esta clasificación ya que es un factor evaluable en el estudio preoperatorio del paciente electivo.

\section{Conclusiones}

La elevada tasa de complicaciones observada en los pacientes octogenarios, frecuentemente asociadas a la descompensación de patologías crónicas de base, se relaciona con mayores tiempos de hospitalización y mayor uso de unidad de pacientes críticos. Concordante con lo anterior, en nuestro trabajo, los pacientes complicados, reoperados o que requirieron hospitalización en UCI o Intermedio, mostraron costos de hospitalización 3 veces mayores que el resto de la serie. Al igual que otras publicaciones, en nuestra serie la edad por sí misma no parece ser un factor de riesgo independiente para morbimortalidad post operatoria, sino que se relaciona más a la presencia de comorbilidades, el grado de compensación de éstas al momento de la cirugía y la necesidad de cirugía de urgencia.

\section{Referencias}

1. Instituto Nacional de Estadísticas (INE). Estadísticas vitales proyección 2005-2050.

2. Turrentine F, Wang H, Simpson V, Jones R. Surgical risk factors, morbidity, and mortality in elderly patients. $\mathrm{J}$ 
Am Coll Surg 2006; 203: 865-877.

3. McNicol L, Story D, Leslie K, Myles P, Fink M, Shelton A, et al. Postoperative complications and mortality in older patients having non-cardiac surgery at three Melbourne teaching hospitals. Med J Aust 2007; 186: 447-452.

4. Altamirano C, Catán F, Toledo G, Ormazábal J, Lagos $\mathrm{C}$, Bonacic M, y col. Cirugía abdominal de urgencia en el adulto mayor. Rev Chil Cir 2002; 54: 654-657.

5. Hamel M, Henderson W, Khuri S, Daley J. Surgical outcomes for patients aged 80 and older: morbidity and mortality from major noncardiac surgery. J Am Geriatr Soc 2005; 53: 424-429.

6. Arenal J, Bengoechea-Beeby M. Mortality associated with emergency abdominal surgery in the elderly. Can J Surg 2003; 46: 111-116.

7. Leung JM, Dzankic S. Relative importance of preoperative health status versus intraoperative factors in predicting postoperative adverse outcomes in geriatric surgical patients. J Am Geriatr Soc 2001; 49: 1080-1085. 